\title{
Protein Kinase C
}

National Cancer Institute

\section{Source}

National Cancer Institute. Protein Kinase C. NCI Thesaurus. Code C17017.

A family of serine/threonine protein kinases that have a structurally conserved catalytic subunit and a regulatory subunit that are connected by a hinge domain. 